\title{
Unusual Case of Bilateral Breast Cancer: A Pure Encapsulated Papillary Breast Tumor of the Right Breast and a Contralateral Invasive Ductal Carcinoma
}

\author{
Alberto Testori'1, Valentina Errico', Edoardo Bottoni' ${ }^{1}$, Emanuele Voulaz' ${ }^{1}$, Stefano Meroni2, \\ Roberto Travaglini' ${ }^{1}$, Marco Alloisio ${ }^{1}$ \\ ${ }^{1}$ General and Thoracic Surgery, IRCCS Humanitas Research Hospital, Via Manzoni, Rozzano (Milan), Italy \\ ${ }^{2}$ Breast Imaging Division, European Institute of Oncology, Via Ripamonti, Milan, Italy \\ Email: stefano.meroni@ieo.it
}

Received 9 February 2015; accepted 27 March 2015; published 30 March 2015

Copyright (C) 2015 by authors and Scientific Research Publishing Inc.

This work is licensed under the Creative Commons Attribution International License (CC BY).

http://creativecommons.org/licenses/by/4.0/

(c) (i) Open Access

\begin{abstract}
Background: Intracystic papillary breast cancer is a very rare tumor that occurs most frequently in elderly postmenopausal women. Aim: In this article we presented a case of a 66-year-old woman who underwent excisional biopsy due to a right breast mass. Case presentation: Histological examination revealed the "pure" encapsulated papillary breast carcinoma without coexisting in situ neoplasm and/or invasive carcinoma. This is a rare lesion of the breast that can clinically mimic breast benign mass with only local or regionally aggressive course. Conclusion: In order to avoid misdiagnosis, both the clinician and the breast radiologist should have the possibility of diagnosing this tumor. Intracystic papillary carcinoma of the breast associated with lymph node metastasis has rarely been reported, but the sentinel lymph node biopsy may be prudent in such cases, despite the non aggressive behavior.
\end{abstract}

\section{Keywords}

Encapsulated Papillary Breast Carcinoma, Intracystic Papillary Breast Carcinoma, Lymph Node Breast Metastases

\section{Introduction}

Intracystic papillary breast cancer is a ductal carcinoma of papillary variety surrounded by a fibrous capsule that

How to cite this paper: Testori, A., et al. (2015) Unusual Case of Bilateral Breast Cancer: A Pure Encapsulated Papillary Breast Tumor of the Right Breast and a Contralateral Invasive Ductal Carcinoma. Advances in Breast Cancer Research, 4, 3943. http://dx.doi.org/10.4236/abcr.2015.42004 
develops in a cystic space. It occurs most frequently in elderly postmenopausal women.

It is a rare clinicopathological entity, and its in situ or invasive character is difficult to establish, particularly on biopsy. Surgery and breast conservation are treatment options, depending on the size of the tumor. This tumor may be multifocal and can be as a "pure" form or associated with in situ neoplasms or invasive carcinomas. Lymph node exploration is currently debated. Although rare and despite being a large and bulky tumor, invasive papillary cancer has an excellent prognosis [1] due to a high-grade hormonal response and uncommon axillary node metastases. The prognosis is favorable also for women presenting with axillary metastases [2].

The aim of this article is to present a case of unexpected bilateral breast cancer and to make breast radiologists and surgeons aware of the spread of the axillary tumor.

\section{Clinical Summary}

We report a case of a 66-year-old female presenting with a painless mass in the upper inner quadrant of the right breast.

On physical examination the breast surgeon appreciated a rather firm palpable lump without any evidence of dermal invasion and no suspicious axillary lymph nodes. A round, high-density mass without microcalcifications, with well-defined margins and $15 \mathrm{~mm}$ in diameter was found in the right breast on a mammography (Figure 1). An ultrasound examination revealed one well-delimited oval mass with a slightly heterogeneous echostructure. The nodule featured cystic collections in hypoechoic solid parts containing millimetric cystic regions. There was no acoustic shadowing; a slight acoustic enhancement behind the mass was noticed instead and there were no sonographic signs of adjacent tissue invasion (Figure 2). No suspicious axillary lymph nodes were found on ultrasound examination. The breast ecographic findings did not raise any suspicion of malignancy: the radiological work-up was rather suggestive of a benign etiology such as pseudoangiomatous stromal hyperplasia (PASH), fibroadenoma, intraductal papilloma, or complex cyst. In contrast, rare malignant tumors including medullary or mucinous carcinoma were considered as well. The serum CEA and CA15-3 levels were within normal range. A fine needle aspiration cytology (FNAC) under ultrasound guidance of the nodule was performed and revealed a C3 diagnosis, most likely a benign lesion. Lumpectomy was performed in another medical center; no surgical margin was positive for malignancy.

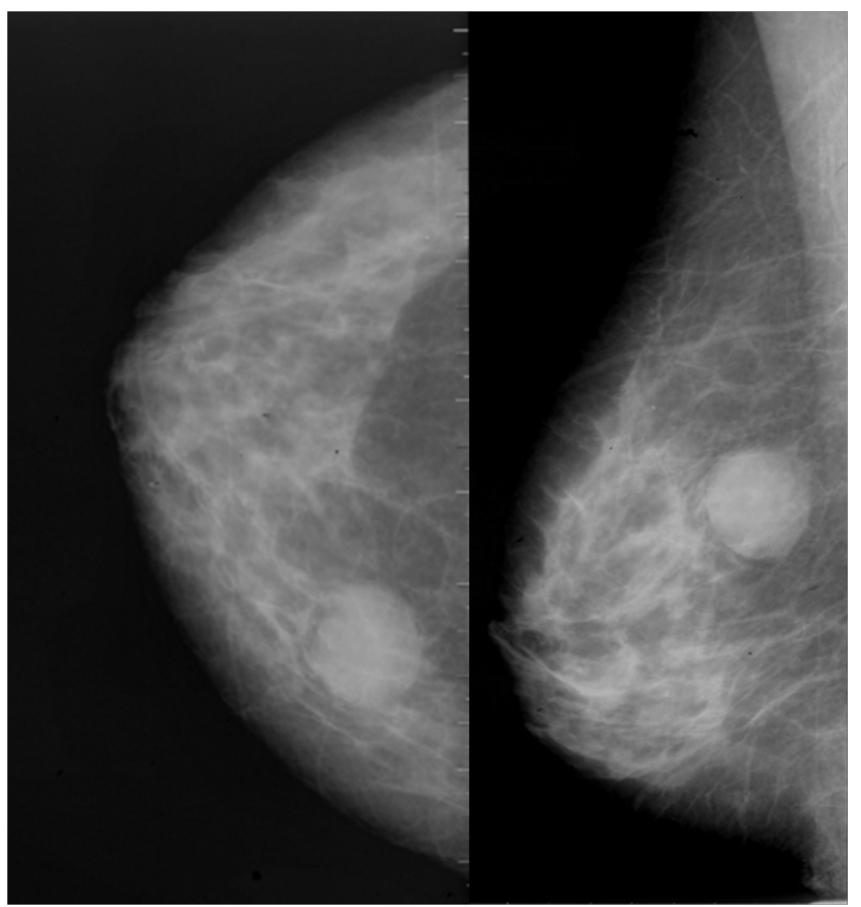

Figure 1. Mediolateral oblique and craniocaudal mammograms of the right breast showing a high opacity round mass without microcalcification and with well-defined margins in the upper inner quadrant. 


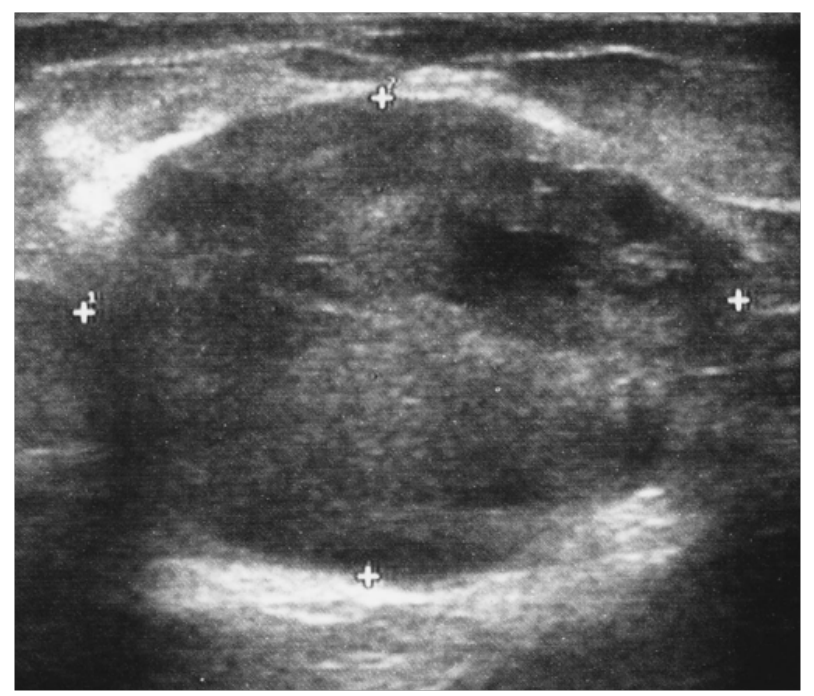

Figure 2. Ultrasound imaging demonstrating a round mass with well-defined margins and a slight acoustic enhancement behind the mass. Multiple areas of hypoechogenicity are detectable, revealing cystic areas.

Microscopic evaluation revealed a "pure" encapsulated papillary breast carcinoma without coexisting in situ neoplasm (ER 90\%, PgR 90\%, Ki67 30\% Her2 negative).

The patient was referred to our institution for sentinel lymph node biopsy. Sentinel lymph node metastasis was detected in the right axilla using SNOLL (Sentinel Node and Occult Lesion Localization) procedure. We performed a lymph node dissection of the right axilla. Due to axillary tumor spread, the patient underwent breast MRI (Magnetic Resonance Imaging), which showed a small suspicious nodule in the left breast undetected with the traditionally triplet diagnostics (physical examination, mammography and ultrasound).

At the breast MR imaging, the suspicious nodule showed an ill-defined mass without a cystic component, characterized by hypointensity at axial $\mathrm{T} 2$-weighted image, hypointensity at the T1-weighted imaging findings and hyperintensity after administration of gadolinium-based contrasting agent. This finding represented the vascularized stroma.

The dynamic enhancement pattern is typically the type III washout pattern in the solid portion.

So we performed a left quadrantectomy with sentinel node biopsy using SNOLL procedure without a subsequent axillary dissection. In the left breast, the final diagnosis was invasive ductal carcinoma not otherwise specified, G2 (ER 90\%, PgR 75\%, Ki67 10\% Her2 negative), with isolated tumor cells in one lymph node.

According to the biological characteristics of ductal breast cancer, we proposed radiation therapy on both breasts and hormone therapy.

An annual mammography and a six month breast ultrasound with senological examination were scheduled, considering the malignant nature of the lesion in a right breast. After two years of follow-up, we found no recurrence.

\section{Discussion}

The aim of this article is to highlight an uncommon case of encapsulated papillary breast cancer with an atypical clinical presentation [1] [3]-[6]. Imaging is crucial for the identification of the lesion and local staging, guiding tissue diagnosis, and follow-up while the final diagnosis still relies on the interventional approach (biopsy or surgery). Considering the radiological features of the right breast mass (rounded shape, well-defined margins, heterogeneous echostructure, absence of acoustic shadowing, absence of microcalcifications and an expansive rather than infiltrative growth pattern) the diagnosis of a benign or probably benign lesion could be erroneously made.

Differentiation between benign and malignant papillary lesions may be difficult on FNAC (as in our case), because FNAC targets the centre whereas invasion is at the periphery, as well as the trucut biopsy of the lesion. Despite the fact that these tumors are invasive or in situ neoplasms, encapsulated papillary breast carcinomas 
rarely metastasize, and when they do, they must be considered as indistinguishable from general mammary carcinomas.

Based on the available clinical data [3]-[6], little is known about encapsulated papillary breast cancer with positive axillary lymph node [2]. Axillary dissection is rare because nodal metastases are poor in this group of patients, however sentinel node biopsy may be warranted. Our case indicates that some papillary breast cancers can widely spread and sentinel lymph node biopsy may be a prudent way to evaluate axillary involvement in these patients. It is difficult to make a correct preoperative evaluation in such cases but, in our opinion, the sentinel lymph node biopsy is a reasonable option. Since encapsulated papillary breast carcinomas rarely metastasize, when we found metastases in the sentinel node, we decided to reassess the initial diagnosis by subjecting the patient to a breast MRI with contrasting agents. Interestingly, using MRI to detect an eventual another breast cancer in a right breast, we found an otherwise occult contralateral breast cancer not detected with traditional diagnostic triplet (physical examination, mammography and ultrasound).

Invasive papillary carcinoma often has a favorable prognosis than ductal carcinoma. In a recent study [7], the majority of invasive papillary carcinoma patients were positive for ER and PgR and negative for Her2 (as in our case), low-grade and slow growing cancer. The incidences of local recurrence, distant metastasis, and cancer related death were relatively low to that previously reported [6]-[8]. Surgical resection remains the cornerstone of treatment with an undefined role for radiation therapy and chemotherapy in the neoadjuvant, adjuvant, and metastatic management [6]-[8]. The aim of this article is to present a case report of encapsulated breast cancer with axillary node metastasis tumor and to make the clinician and the radiologist aware of the possible axillary tumor spread of these tumors. The second aim of this report is to raise the radiologist awareness towards ultrasound abnormalities in breast nodules and to evaluate accurately the axillary node in case of breast lesion with atypical clinical and radiological presentations.

\section{Conclusion}

In conclusion, we demonstrate a case of invasive papillary carcinoma of the breast with omolateral axillary tumor spread, rare by definition and an undetected tumor on the left side. Recommendation from this case report is to have a great attention on benign diagnosis. Make an adequate follow-up also for benign lesions as pseudoangiomatous stromal hyperplasia (PASH), fibroadenoma, intraductal papilloma, or complex cyst to evaluate a possible variation, and perform MRI in all doubt or uncommon cases.

\section{Disclosure Statement}

This research was not supported by any organization and none of the authors has a financial relationship that would represent a conflict of interest. The informed consent was obtained by the patient.

\section{References}

[1] Rakha, E.A., Gandhi, N., Climent, F., van Deurzen, C.H., Haider, S.A., Dunk, L., Lee, A.H., Macmillan, D. and Ellis, I.O. (2011) Encapsulated Papillary Carcinoma of the Breast: An Invasive Tumor with Excellent Prognosis. American Journal of Surgical Pathology, 35, 1093-1103. http://dx.doi.org/10.1097/PAS.0b013e31821b3f65

[2] Mulligan, A.M. and O’Malley, F.P. (2007) Metastatic Potential of Encapsulated (Intracystic) Papillary Carcinoma of the Breast: A Report of 2 Cases with Axillary Lymph Node Micrometastases. International Journal of Surgical Pathology, 15, 143-147. http://dx.doi.org/10.1177/1066896906299119

[3] Rodríguez, M.C., Secades, A.L. and Angulo, J.M. (2010) Best Cases from the AFIP: Intracystic Papillary Carcinoma of the Breast. Radiographics, 30, 2021-2027. http://dx.doi.org/10.1148/rg.307105003

[4] Dogan, B.E., Whitman, G.J., Middleton, L.P. and Phelps, M. (2003) Intracystic Papillary Carcinoma of the Breast. American Journal of Roentgenology, 181, 186. http://dx.doi.org/10.2214/ajr.181.1.1810186

[5] Akagi, T., Kinoshita, T., Shien, T., Hojo, T., Akashi-Tanaka, S. and Murata, Y. (2009) Clinical and Pathological Features of Intracystic Papillary Carcinoma of the Breast. Surgery Today, 39, 5-8. http://dx.doi.org/10.1007/s00595-008-3792-9

[6] Grabowski, J., Salzstein, S.L., Sadler, G.R. and Blair, S. (2008) Intracystic Papillary Carcinoma: A Review of 917 Cases. Cancer, 113, 916-920. http://dx.doi.org/10.1002/cncr.23723

[7] Liu, Z.Y., Liu, N., Wang, Y.H., Yang, C.C., Zhang, J., Ly, S.H. and Niu, Y. (2013) Clinicopathologic Characteristics and Molecular Subtypes of Invasive Papillary Carcinoma of the Breast: A Large Case Study. Journal of Cancer Re- 
search and Clinical Oncology, 139, 77-84. http://dx.doi.org/10.1007/s00432-012-1302-3

[8] Solorzano, C.C., Middleton, L.P., Hunt, K.K., Mirza, N., Meric, F., Kuerer, H.M., Ross, M.I., Ames, F.C., Feig, B.W., Pollock, R.E., Singletary, S.E. and Babiera, G. (2002) Treatment and Outcome of Patients with Intracystic Papillary Carcinoma of the Breast. The American Journal of Surgery, 184, 364-368.

http://dx.doi.org/10.1016/S0002-9610(02)00941-8 